\title{
Grain-boundary engineering markedly reduces susceptibility to intergranular hydrogen embrittlement in metallic materials
}

\author{
S. Bechtle, ${ }^{1,2}$ M. Kumar, ${ }^{3}$ B. P. Somerday, ${ }^{4}$ M. E. Launey ${ }^{5}$ and R. O. Ritchie ${ }^{1,5^{*}}$ \\ ${ }^{1}$ Department of Materials Science \& Engineering, University of California, Berkeley, CA 94720, USA \\ 2Technical University of Hamburg-Harburg, DE-21073 Hamburg, Germany \\ ${ }^{3}$ Lawrence Livermore National Laboratory, Livermore, CA 94550, USA \\ ${ }^{4}$ Sandia National Laboratories, Livermore, CA 94551, USA \\ ${ }^{5}$ Materials Sciences Division, Lawrence Berkeley National Laboratory, Berkeley, CA 94720, USA
}

\begin{abstract}
The feasibility of using "grain-boundary engineering" techniques to reduce the susceptibility of a metallic material to intergranular embrittlement in the presence of hydrogen is examined. Using thermomechanical processing, the fraction of "special" grain boundaries was increased from 46 to $75 \%$ (by length) in commercially purity Nickel samples. In the presence of hydrogen concentrations between 1200 and 3400 appm, the high special fraction microstructure showed almost double the tensile ductility; also, the proportion of intergranular fracture was significantly lower and the $J_{c}$ fracture toughness values were some 20 to $30 \%$ higher in comparison with the low special fraction microstructure. We attribute the reduction in the severity of hydrogen-induced intergranular embrittlement to the higher fraction of special grain boundaries which reduces the crack initiation site density.
\end{abstract}

Keywords: Hydrogen embrittlement; Intergranular cracking; Grain-boundary engineering; Special boundaries; Mechanical properties

\footnotetext{
* Contributing author - email: roritchie@lbl.gov; tel. (510) 486-5798
} 


\section{Introduction}

Few can deny the importance of hydrogen as a clean, mobile energy carrier and source of fuel, whether as a power source for automobiles or transported in highpressure $(\sim 20-100 \mathrm{MPa})$ pipelines. However, the problems associated with hydrogen storage and containment are substantial. Of particular significance is the potential degradation of metallic containment systems, principally steel pressure vessels, tanks and pipelines, where hydrogen uptake in the metal can induce premature, unexpected and potentially catastrophic failures [1]. Hydrogen, either as an external gas, resulting from electrochemical reactions in aqueous environments or dissolved in the metal, is known to markedly lower the ductility, fracture strength and fracture toughness, and to accelerate subcritical cracking under sustained and/or cyclic loading [2]. The mechanisms associated with such structural degradation have remained an issue of contention for many years, but can be broadly classified into three primary mechanisms, namely decohesion mechanisms, where hydrogen at internal interfaces lowers the cohesive strength ("hydrogen embrittlement"1), hydrogen-enhanced local plasticity, where hydrogen affects the local instabilities associated with plastic flow, and in certain materials, e.g., $\mathrm{Ti}$ and $\mathrm{Zr}$, hydride formation, where the presence of highly brittle hydride precipitates results in a "low energy" fracture path [3]. In addition, there are other hydrogen-related degradation mechanisms involving precipitation of internal gaseous species at higher pressures and temperatures, such as blistering and hydrogen attack.

Despite the many types of hydrogen-induced degradation mechanisms, the most devastating is hydrogen embrittlement which in many high-strength metals and alloys results in a sharp transition from a high-toughness ductile (microvoid coalescence)

\footnotetext{
${ }^{1}$ We use the term "hydrogen embrittlement" in this paper specifically to refer to the degradation in mechanical properties caused by the presence of hydrogen that results in intergranular embrittlement.
} 
fracture to a low-toughness brittle fracture with an associated dramatic loss in ductility, strength and toughness [4]. In metallic materials such as high-strength steels and nickel, etc., this embrittlement is manifest as a loss of cohesion primarily in the grain boundaries, leading to low toughness intergranular fracture. Due to its rapid diffusion, hydrogen ingress is difficult to stop; all forms of the resulting degradation in mechanical properties are of concern, but it is this intergranular embrittlement that is the most serious as it can induce time-dependent subcritical cracking at low applied stresses, which can lead to unexpected catastrophic failures of structures. The effect is generally considered to result from one of two scenarios: (i) hydrogen in the metal diffuses to the grain boundaries where it lowers intergranular cohesion, or (ii) alloying/impurity elements, e.g., $\mathrm{Mn}, \mathrm{Si}, \mathrm{S}$ and $\mathrm{P}$, segregate to the boundaries, aiding decohesion-the resulting intergranular crack attracts hydrogen which further accelerates the fracture [4]. Whatever the mechanism, the crystallographic character of the grain boundaries is of paramount importance as it is here that the solute element segregants and/or hydrogen cause the embrittlement.

Our approach in this work is to attempt to eliminate, or at least significantly reduce, the severity of hydrogen embrittlement by designing microstructures with networks of lower energy grain boundaries (so called "special" boundaries, such as annealing twins and their variants) that are less likely to be preferred sites for segregants and that are inherently more resistant to intergranular fracture. For instance, it has been shown for bismuth or sulfur segregation in copper [5-7], that compared to random boundaries, the special boundaries are only partially covered by the segregating species; it is also known that such coverage can have a marked influence on macroscopic ductility [8]. Using a model material of commercially pure nickel (Nickel-201), we achieve this using the notion of grain-boundary engineering, where we employ thermomechanical processing to promote the nucleation of such special boundaries, which 
correspondingly reduce the incidence of high-energy "random" grain boundaries in the microstructure. Indeed, it has been claimed that this technique can yield improvements in strength, ductility, chemical corrosion, stress-corrosion cracking, high-temperature creep and high-cycle fatigue resistance [9-13]. Our hypothesis here is that any enhancement in the special boundary fraction, which will concomitantly reduce the connectivity of the random grain-boundary network [14-17] will lower the incidence of intergranular fracture in the presence of hydrogen such that high-energy ductile transgranular fracture will ensue.

"Special" grain boundaries are characterized by a particular misorientation, low excess free volumes and a high degree of atomic matching; they are described geometrically by a low "sigma number", $\Sigma(1<\Sigma \leq 29)$, which is defined in terms of the coincident-site lattice (CSL) model as the reciprocal of the fraction of lattice points in the boundaries that coincide between the two adjoining grains, with an allowable angular deviation from the Brandon criterion of $\Delta \theta \leq 15^{\circ} \cdot \Sigma^{-1 / 2}$ [18]. Statistically significant grain orientation maps of microstructures can be obtained using SEM-based electron backscatter diffraction (EBSD) methods [19]. Processing generally involves several strain-annealing cycles to induce strain by cold working and strain-induced grainboundary migration during subsequent annealing, the latter creating special grain boundaries via a boundary decomposition mechanism in the grain-boundary network [20]. In addition to an enhanced fraction of special boundaries, such "engineered" microstructures can possess a refined grain size and diminished incidence of deviation from the exact $\Sigma$ misorientations; the texture, however, generally remains unchanged or, in some cases, can be reduced.

Accordingly, in this work we examine the effect of internal hydrogen concentration on the mechanical properties and fractography of commercial nickel with microstructures "engineered" to have low and high special boundary fractions. Our 
results show that the susceptibility to intergranular embrittlement, defined in terms of the tensile ductility, fracture toughness and incidence of intergranular fracture, is definitively reduced in microstructures with a higher proportion of special grain boundaries.

\section{Experimental Methods}

\subsection{Materials}

Investigations were conducted on a Nickel-201 alloy, a commercially pure nickel supplied by ThyssenKrupp VDM USA, Inc., with impurities (in wt.\%) of $0.11 \mathrm{Mn}, 0.08$ $\mathrm{Fe}, 0.02 \mathrm{Si}, 0.01 \mathrm{Cu}, 0.01 \mathrm{C}$ and $0.002 \mathrm{~S}$. The Ni-201 was received in the form of $25.4 \mathrm{~mm}$ thick plate in the "soft annealed" condition. Using $25 \mathrm{~mm} \mathrm{sq}, 80 \mathrm{~mm}$ long sections, we employed three cycles of cold rolling ( $5 \%$ reduction in thickness per cycle) followed by a $15 \mathrm{~min}$ anneal at $900^{\circ} \mathrm{C}$ in an air furnace (water quenched), to achieve a microstructure with a high length fraction of $75 \%$ special boundaries ( $62 \%$ number fraction). This was compared to a second microstructure which was cold rolled four times ( $20 \%$ reduction in thickness per cycle) followed by a 15 min anneal at $700^{\circ} \mathrm{C}$ in an air furnace (water quenched) with a final $1 \mathrm{hr}$ grain-coarsening anneal at $900^{\circ} \mathrm{C}$, to achieve a low length fraction of $46 \%$ special boundaries ( $35 \%$ number fraction), practically all of which are $\Sigma 3$ twin boundaries. We analyzed these microstructures using EBSD orientation mapping; they are shown in Fig. 1 with random boundaries depicted in black, $\Sigma 3^{n}$ special boundaries in blue, and the $\Sigma 3$ twin boundaries in red. Corresponding characteristics of these microstructures are listed in Table 1. The crystallographic texture (close to that of a random polycrystal) and grain size $(\sim 30 \mu \mathrm{m})$ were independent of the thermomechanical processing.

\subsection{Hydrogen charging}


Evaluation of the mechanical properties was achieved using uniaxial tensile tests and fracture toughness testing on single edge-notched bend specimens. Atomic hydrogen was dissolved into the tensile and bend-bar specimens by thermally charging the specimens in high-pressure hydrogen gas. The specimens were inserted into a thickwalled stainless steel (A-286) pressure vessel, which was located in a furnace. After sealing the pressure vessel, residual gases were removed from the vessel and gas handling manifold using a sequence of purging and evacuating. Three purge/evacuation cycles were conducted with helium, followed by three purge/evacuation cycles with hydrogen. Once the purging/evacuation cycles were completed, the pressure vessel was heated and then filled with $99.9999 \%$ hydrogen gas. Thermal charging was conducted at elevated temperature to enhance hydrogen diffusion (minimize charging times), but temperatures were limited to avoid thermallyactivated microstructure changes in the nickel. Diffusion calculations showed that a uniform hydrogen concentration could be attained in the $4 \mathrm{~mm}$-diameter tensile specimen in $120 \mathrm{hr}$ by charging at a minimum temperature of $150^{\circ} \mathrm{C}$. The bend-bar specimens had larger dimensions (10 $\mathrm{mm}$ by $10 \mathrm{~mm}$ cross section), so the charging temperature was increased to at least $200^{\circ} \mathrm{C}$ to enhance hydrogen diffusion. For these specimen dimensions and charging temperature, a uniform hydrogen concentration was established after $280 \mathrm{hr}$.

At each charging temperature, hydrogen gas pressures were selected to yield lattice hydrogen concentrations in the nickel specimens of 1000, 2000 or $3000 \mathrm{appm}$. The extent of hydrogen-induced intergranular fracture in high-purity nickel depends on the lattice hydrogen concentration, and the range of hydrogen concentrations selected for this study was expected to produce predominantly intergranular fracture [21]. The hydrogen fugacity needed to produce a particular equilibrium lattice hydrogen concentration was calculated from the following expressions: 


$$
\begin{gathered}
C_{L}=S f^{1 / 2}, \\
S=S_{0} \exp \left(\frac{-H_{S}}{R T}\right),
\end{gathered}
$$

where $C_{L}$ is the lattice hydrogen concentration, $f$ is the fugacity of the gas, $S$ is the solubility, $H_{s}$ is the heat of solution, and $R$ and $T$ are the gas constant and absolute temperature, respectively. Parameters in the relationship for the solubility of hydrogen in nickel (Eq. 2) were reported as $S_{0}=9.7 \times 10^{3} \mathrm{appm} \cdot(\mathrm{MPa})^{-1 / 2}$ and $H_{S}=15.9 \mathrm{~kJ} \cdot \mathrm{mol}^{-1}[22$, 23]. With the exposure temperature and target hydrogen concentration fixed, the required gas fugacity was calculated from Eqs. 1-2. From the calculated fugacity, the gas pressure to be used during hydrogen charging was determined from the following expression [24]:

$$
f=P \exp \left(\frac{P b}{R T}\right)
$$

where $P$ is the pressure and $b$ is a constant in the Abel-Noble equation of state. The combinations of temperature and gas pressure used to produce specific target lattice hydrogen concentrations are summarized in Table 2.

The actual total hydrogen concentrations in the tensile and single edge-notched bend specimens were established through measurements. Small pieces were sectioned from the tensile and single edge-notched bend specimens after hydrogen exposure and mechanical testing and sent to Wah Chang (Albany, OR) for analysis. The hydrogen concentration was measured using an inert gas-fusion instrument. For most hydrogen pressure/temperature combinations, measured total hydrogen concentrations were $\sim 10$ to $20 \%$ greater than the calculated lattice concentrations (Table 2). The exception was one set of bend specimens, where the measured hydrogen concentration was nearly $50 \%$ greater than the target concentration. It is expected that actual concentrations can exceed calculated values, since dissolved atomic hydrogen fills sites not only in the 
metal lattice but also at defects (e.g., grain boundaries and dislocations). When the metal-hydrogen system reaches equilibrium in the hydrogen pressure/temperature environment, the lattice hydrogen concentration is accurately represented by Eqs. 1-2 but the hydrogen at defects ("trapped" hydrogen) is not included. The measurements yield the total hydrogen concentration in the lattice and trap sites that were developed at equilibrium during hydrogen charging.

Since the thermal charging of specimens was conducted in a thick-walled pressure vessel, the vessel cooled slowly to room temperature after switching off power to the furnace. During this cooling period of $\sim 16 \mathrm{hr}$, hydrogen egress from the specimens occurred. Based on the estimated temperature and gas pressure vs. time history for the specimens, diffusion calculations were conducted to assess the extent of hydrogen egress. These calculations demonstrated that the resulting hydrogen concentration gradient was limited to a near-surface region of $\sim 10 \%$ of the total hydrogen penetration distance.

\subsection{Segregation studies}

The possibility of solute segregation to grain boundaries, in particular sulfur, was studied using an Auger spectroscopy analysis of intergranular fracture facets. Notched specimens of Ni-201 were charged with hydrogen to a target lattice concentration of 2000 appm, and the specimens were fractured in the Auger chamber to expose intergranular facets, which were then examined for the presence of solute elements.

\subsection{Mechanical testing}

\subsubsection{Uniaxial tensile testing}

Uniaxial tensile tests were conducted at room temperature using 4-mm diameter round uniaxial tensile specimens with $25 \mathrm{~mm}$ gauge length, oriented lengthwise 
parallel to the rolling direction. These were machined from the thermomechanically treated material and loaded, according to ASTM Standard E8 M-08 [25], on a servohydraulic MTS 810 mechanical testing machine (MTS Corporation, Eden Prairie, MN), operating in displacement control at a displacement rate of $0.01 \mathrm{~mm} / \mathrm{sec}$. This relatively high displacement rate was selected to minimize hydrogen egress from the hydrogencharged Ni-201 by limiting the duration of the tests. Although lower displacement rates are often employed in testing of hydrogen-charged specimens to allow time for diffusion-controlled hydrogen redistribution to fracture sites, studies of hydrogencharged, high-purity nickel demonstrated that hydrogen redistribution during straining was not necessary to promote intergranular embrittlement $[21,26]$.

Ductilities were determined from measurements of the reduction in area, RA:

$$
\mathrm{RA}=\left(d_{\mathrm{i}}{ }^{2}-d_{\mathrm{r}}{ }^{2}\right) / d^{2}{ }^{2},
$$

where $d_{\mathrm{i}}$ is the initial specimen diameter before testing, and $d_{\mathrm{f}}$ is the final diameter after fracture.

\subsubsection{R-curve fracture toughness testing}

Evaluation of the fracture toughness was obtained from J-R curves generated using 10-mm thick, single-edge notched bend (SE(B)) specimens, also oriented lengthwise parallel to the rolling direction. Prior to J-R curve testing, the SE(B) specimens were pre-cracked in four-point bending using a servo-hydraulic MTS 810 mechanical testing machine (MTS Corp., Eden Prairie, MN). Loads were transferred to the specimen using a bend fixture equipped with $6.3 \mathrm{~mm}$ diameter roller supports. Pre-cracks were grown from an initial machined notch length of $0.4 \mathrm{~W}$ to a final crack length of $0.5 \mathrm{~W}$, where $\mathrm{W}$ $(=10 \mathrm{~mm})$ is the width of the sample. A cyclic frequency of $25 \mathrm{~Hz}$ (sine wave) was employed with a constant load ratio (ratio of minimum to maximum load, $R=P_{\min } / P_{\max }$ ) of 0.1 and a final maximum stress intensity of $K_{\max } \sim 8.8 \mathrm{MPa} \sqrt{\mathrm{m}}$. Crack lengths were 
monitored during pre-cracking using unloading elastic compliance measurements from a $350 \Omega$ strain gauge attached to the back face of the specimen. After fatigue precracking, the samples were side-grooved ( $10 \%$ of the thickness each side) to increase the constraint and maintain straight crack fronts during the R-curve tests.

$J-R$ curves were measured to evaluate the resistance to fracture in terms of the $J$ integral as a function of crack extension, $\Delta a$, under a monotonically increasing driving force. Tests were conducted in three-point bending with a loading span $(40 \mathrm{~mm})$ to width $(10 \mathrm{~mm})$ ratio of 4 , in accordance with ASTM E1820-06 [27]. The specimens were loaded in displacement control using a servo-hydraulic MTS 810 testing machine with a loading displacement rate of $\sim 0.01 \mathrm{~mm} / \mathrm{sec}$ until the onset of cracking, which was determined by non-linearity in the load-displacement curve. Following the rationale applied to the tensile tests, a relatively high displacement rate was selected for testing of hydrogen-charged SE(B) specimens since intergranular embrittlement could be induced in nickel without time-dependent hydrogen redistribution during loading. At this point, the sample was periodically unloaded by $10-20 \%$ of the peak load to record the sample load-line compliance at the new crack length using a linear variable-differential transformer (LVDT) mounted in the load frame. After each loading increment, the specimens were held for $30 \mathrm{sec}$ to allow for crack extension to stabilize, followed by unloading compliance measurement. This process was repeated at regular intervals until the end of the test, at which point the compliance and loading data were analyzed to determine $J$-integral as a function of crack extension, $\Delta a$. Crack lengths, $a$, were calculated from the compliance data obtained during the test using compliance expression of a three-point bend specimen at load line for $S / W=4$ [28]:

$$
a / W=0.997-3.58 U-1.51 U^{2}-110 U^{3}+1232 U^{4}-4400 U^{5},
$$

where $U$ is a fitting function, written as : 


$$
U=\frac{1}{\sqrt{F C}+1}
$$

Here $C$ is the sample compliance, and $F$ is a calibration factor, taken to be that which gives the best agreement between the initial compliance and crack length at the beginning of the test.

The stress intensity at each measured crack length was calculated by measuring the nonlinear strain-energy release rate, $J$, where $J$ is defined as the rate of change in potential energy under nonlinear-elastic conditions for a unit increase in crack area, which is equivalent to the strain energy release rate $G$ under elastic conditions. J was calculated from the applied load and instantaneous crack length according to ASTM standard E1820-06 [27], and was decomposed into its elastic and plastic contributions:

$$
J=J_{\mathrm{el}}+J_{\mathrm{pl}}
$$

The elastic contribution $J_{\mathrm{el}}$ is based on linear elastic fracture mechanics:

$$
J_{\mathrm{el}}=\frac{K_{\mathrm{I}}^{2}}{E^{\prime}}
$$

where $K_{\mathrm{I}}$ is the stress intensity factor (in mode $\mathrm{I}$ ) and $E^{\prime}=E$ in plane stress ( $E$ is Young's modulus). For SE(B) specimens, $K_{\mathrm{I}}$ is given by [27]:

$$
K_{\mathrm{I}}=\frac{P S}{\left(B B_{N}\right)^{1 / 2} W^{3 / 2}} f(a / W)
$$

where $P$ is the applied load, $S$ is the span, $B$ is the specimen thickness, $B_{N}$ is the net specimen thickness, $W$ is the width, and $f$ is an non-dimensional function of the crack length $a$ given by [27]:

$$
f(a / W)=\frac{3(a / W)^{1 / 2}\left[1.99-(a / W)(1-a / W)\left(2.15-3.93(a / W)+2.7(a / W)^{2}\right)\right]}{2(1+2 a / W)(1-a / W)^{3 / 2}}
$$


For a stationary crack in this bending configuration, the plastic component $J_{\mathrm{pl}}$ is given by:

$$
J_{\mathrm{pl}}=\frac{1.9 A_{\mathrm{pl}}}{B_{N} b},
$$

where $A_{\mathrm{pl}}$ is the plastic area under the force versus displacement curve, and $b$ is the uncracked ligament length, $b=W-a$. To take in account the fact that the crack is advancing and that the ligament $b$ is continuously decreasing, an incremental definition of Eq. (11) was used [27]:

$$
J_{p l(i)}=\left[J_{p l(i-1)}+\left(\frac{1.9}{b_{(i-1)}}\right)\left(\frac{A_{p l(i)}-A_{p l(i-1)}}{B_{N}}\right)\right]\left[1-\frac{a_{(i)}-a_{(i-1)}}{b_{(i-1)}}\right] .
$$

Crack-initiation fracture toughness $J_{\mathrm{c}}$ values were determined from the R-curves essentially at $\Delta a \rightarrow 0$. Due to the relatively low strength of the Ni-201 alloys, the values quoted in this work were not in plane strain. However, since all R-curves tests were performed with specimens of an identical size and geometry, the approach provides an accurate measure of the relative toughness of the two microstructures.

All values of mechanical properties presented represent at least an average of three measurements per configuration $(N \geq 3)$. Resulting fracture surfaces after both uniaxial tensile and fracture toughness testing were examined in a Hitachi S4300 E/N secondary electron microscope.

\section{Results}

As shown in Table 1, ultimate tensile strengths (UTS) were essentially the same ( $\sim 372-379 \mathrm{MPa})$ in the two as-processed conditions, although with increasing hydrogen uptake UTS values nominally increased in the grain-boundary engineered samples and 
were reduced in the lower special fraction samples. The average yield strengths, however, were significantly increased, from $\sim 55$ to $204 \mathrm{MPa}$, by the higher fraction of special boundaries, though there was virtually no change with hydrogen uptake (Table 1). The increase in flow stress associated with hydrogen in the grain-boundary engineered samples is consistent with observations of increased flow stress in hydrogen-charged austenitic alloys [29]. The contrasting decrease in tensile strength of hydrogen-charged, low special fraction samples is likely due to a truncation of the stress vs. strain response of this material because of the reduced ductility.

From the uniaxial tensile test results, it is apparent that subjecting the $\mathrm{Ni}-201$ to such hydrogen exposures results in a severe loss in tensile ductility (Fig. 2). With hydrogen concentrations between 1200 and 3400 appm, the measured reduction in area ${ }^{2}$ dropped from $74-86 \%$ in the unexposed material, where ductile failure by microvoid coalescence occurred after extensive necking, to $\sim 6-29 \%$ in the hydrogen-charged material, where fracture occurred in a brittle catastrophic fashion. However, of note is that the grainboundary engineered structures displayed a far lower susceptibility to the hydrogen embrittlement. Specifically, the "low special fraction" microstructures had mean reduction in area values of 15, 13 and 7\% for hydrogen concentrations of 1200, 2200 and 3400 appm, respectively (Fig. 2), with fracture occurring by 100\% intergranular cracking (except in the 1200 appm charged structure where it was 70\%) (Figs. 3, 4). In contrast, mean reduction in area values for the grain-boundary engineered "high special fraction" microstructures were 28,22 and $11 \%$ for the same respective hydrogen concentrations (Fig. 2), with much lower fractions of intergranular cracking of 0,40 and 80\%, respectively (Figs. 3, 4).

\footnotetext{
${ }^{2}$ The relative reduction in area, i.e., as normalized by its value for unexposed material, is used here as a quantitative measure of the extent to which embrittlement occurs due to the presence of hydrogen. In addition the fraction of intergranular fracture gives a second measure of the degree of this embrittlement in the grain boundaries.
} 
The important implications from these results are that grain-boundary engineering, involving an increase in the length fraction of special boundaries from 46 to $75 \%$ (35 to $62 \%$ by frequency), can almost double the tensile ductility in the presence of $\sim 1000$ to 2000 appm hydrogen, with an accompanying 60 to 100\% decrease in the fraction of intergranular fracture. With very high hydrogen concentrations of more than 3000 appm, the beneficial effect of the engineered boundaries is subdued, but nevertheless tensile ductilities are still on average $~ 57 \%$ higher in the grain-boundary engineered microstructures, with the fraction of intergranular fracture some $20 \%$ lower. What is also significant about this result is that this markedly enhanced resistance to hydrogen embrittlement is achieved in nominally identical microstructures except for the higher special boundary fraction; furthermore, the more resistant microstructure has a higher yield strength, whereas it is normally the case [2] that higher yield strengths are associated with a reduced resistance to embrittlement.

The beneficial role of the high fraction of special boundaries in enhancing resistance to hydrogen-induced intergranular embrittlement was further apparent in measurements of the fracture toughness and subcritical crack-growth resistance, which we evaluated using nonlinear elastic fracture mechanics by determining crackresistance (J-R) curves. Such J-R curves results for both hydrogen-charged structures are shown in Fig. 5 in terms of the equivalent $J$-integral as a function of crack extension, $\Delta a$, for both the low and high special boundary fraction microstructures. These results clearly indicate that the (crack-initiation) fracture toughness $J_{c}$ is 20 to $30 \%$ higher in the microstructure with the higher fraction of special boundaries. Moreover, the enhanced hydrogen embrittlement resistance of this microstructure is further evidenced by the fact almost two times more stable (subcritical) crack extension occurs prior to catastrophic failure, consistent with the fact that the fracture is not $100 \%$ intergranular as in the case of the low special fraction microstructure (Fig. 6). 


\section{Discussion}

The results from this study clearly demonstrate that hydrogen promotes intergranular embrittlement in nickel, but it is known that such embrittlement can be exacerbated by sulfur that segregates to grain boundaries [26]. It has been suggested that sulfur has two possible roles in aggravating intergranular fracture [26]: sulfur intrinsically embrittles the grain-boundary structure, and sulfur attracts hydrogen to the grain boundary, thereby enhancing embrittlement due to hydrogen. Since grainboundary character can affect the degree of solute segregation to the boundary, interpretation of the correlation between increased fraction of special grain boundaries and reduced intergranular embrittlement in hydrogen-charged Ni-201 must consider segregated sulfur. This issue was addressed here by conducting an Auger spectroscopy analysis of intergranular fracture facets. The Auger analysis did not reveal any sulfur on the intergranular fracture facets, so it was concluded that sulfur did not contribute to intergranular embrittlement in the Ni-201. The conclusion that segregated sulfur was not necessary for intergranular embrittlement in hydrogen-charged Ni-201 is consistent with results from other studies on hydrogen-charged nickel [26].

The benefit of special grain boundaries in Ni-201 can be interpreted based on a criterion for intergranular embrittlement, i.e., the grain-boundary hydrogen concentration must exceed a critical level for boundary fracture. For a given type of grain boundary, e.g., special or random, the critical hydrogen concentration is not a constant value at each boundary in the microstructure but rather depends on the resolved remote stress and any local stress concentration at individual boundaries [21]. Fracture is favored at grain boundaries with the lowest critical hydrogen concentrations. Studies of hydrogen-charged, high-purity nickel demonstrated that the hydrogen concentration at grain boundaries must be enhanced through segregation to 
attain the critical hydrogen concentration for fracture [21,26,30]. The degree of hydrogen segregation to grain boundaries is estimated from the following relationship [31]:

$$
C_{B} \approx C_{L} \exp \left(\frac{E_{B}}{k T}\right)
$$

where $C_{B}$ is the grain-boundary hydrogen concentration, $C_{L}$ is the lattice hydrogen concentration, $E_{B}$ is the binding energy of hydrogen to the grain boundary, $k$ is Boltzmann's constant and $T$ is absolute temperature.

An effect of special grain boundaries on either element of the fracture criterion (grain boundary hydrogen concentration or critical hydrogen concentration for fracture) can be invoked to rationalize the data in Figs. 2 and 3. It can be assumed that special boundaries are intrinsically more resistant to fracture than random boundaries, and thus the critical hydrogen concentration for grain-boundary fracture is higher for special boundaries. This view follows from assuming that $C_{B} / C_{L}$ is the same for special and random boundaries, i.e., $E_{B}$ is the same for both types of boundaries (Eq. 13). More extensive intergranular fracture in the material with the low special boundary fraction (Fig. 3) is then due to the lower critical hydrogen concentration for fracture at random boundaries. An alternate possibility is that hydrogen has a higher binding energy, $E_{B}$, at random boundaries as compared to special boundaries. In this case, $C_{B} / C_{L}$ is greater for the material with fewer special boundaries, and these higher grain boundary hydrogen concentrations lead to higher proportions of intergranular fracture.

Although the data in Fig. 3 can be rationalized based on two possible effects of special boundaries on the fracture criterion, their effect on hydrogen segregation may dominate. Figure 3 shows that at a total measured hydrogen concentration of 3400 appm, both the low and high special boundary fraction materials exhibit nearly $100 \%$ intergranular fracture. When the fracture mode is solely intergranular, the normalized 
reduction in area values provide a metric for the vulnerability of grain boundaries to hydrogen-induced degradation. Since relative reduction in area values at $3400 \mathrm{appm}$ hydrogen are comparable for the two materials (Fig. 2), grain boundaries in both materials may be similarly vulnerable to hydrogen-induced degradation, i.e., the critical hydrogen concentrations may be similar. This may be reasonable if degradation is not governed by hydrogen interactions with the grain-boundary interface. For example, hydrogen effects on plastic deformation in material immediately adjacent to the grainboundary interface may contribute to intergranular fracture, as suggested for highpurity nickel $[21,26]$. The evidence presented for plasticity-mediated intergranular fracture in hydrogen-charged nickel includes the observation of slip traces on intergranular fracture facets $[21,26]$. Such slip traces appear on intergranular fracture facets from the hydrogen-charged Ni-201 (Fig. 7), supporting the notion that plastic deformation plays a role in intergranular fracture.

\section{Conclusions}

Taken together, these results are a clear indication of the utility of increasing the fraction of special grain boundaries by grain-boundary engineering to markedly enhance resistance to hydrogen-induced intergranular embrittlement in metallic materials, specifically Nickel-201 in the present study. Specific conclusions are:

1. For dissolved hydrogen concentrations of between 1200 and 3400 appm, microstructures comprising a higher (length) fraction (75\%) of special (principally twin) boundaries displayed almost twice the tensile ductility and 20-30\% higher fracture toughness, as compared to microstructures containing much lower (46\%) special fractions, consistent with a lower proportion of intergranular fracture.

2. In specific $J-R$ resistance-curve measurements, in addition to the $20-30 \%$ increase in crack-initiation toughness, the extent of stable crack extension prior to catastrophic 
3. It is concluded that the "engineered" microstructures are still embrittled by the presence of hydrogen but the severity of this embrittlement is significantly reduced, whether assessed in terms of the tensile ductility, fracture toughness or fraction of intergranular fracture.

\section{Acknowledgements}

This work was supported by the U.S. Department of Energy: at the Lawrence Berkeley National Laboratory by the Office of Science, Office of Basic Energy Sciences, Division of Materials Sciences and Engineering under Contract No. DE-AC02-05CH11231, at the Lawrence Livermore National Laboratory under Contract No. DE-AC52-07NA27344, and at Sandia National Laboratories under Contract No. DE-AC04-94AL85000.

\section{References}

[1] Irani RS. MRS Bull. 2002;27:680.

[2] Gangloff RP. In: Milne I, Ritchie RO, Karihaloo B, editors. Comprehensive Structural Integrity: Fracture of Materials from Nano to Macro, vol. 6, Elsevier Science, New York, NY 2003. p.31.

[3] Birnbaum HK. MRS Bull. 2003;28:479.

[4] McMahon CJ. Eng. Fract. Mech. 2001;68:773.

[5] Bruley J, Keast VJ, Williams DB. Acta Mater. 1999;47:4009.

[6] Keast VJ, Bruley J, Rez P, Maclaren JM, Williams DB. Acta Mater. 1998;46:481.

[7] Keast VJ, Williams DB. Acta Mater. 1999;47:3999.

[8] Schwartz AJ, Kumar M, Lassila DH. Metall. Mater. Trans. A 2004;35A:2567.

[9] Palumbo G, Aust KT. Scripta Metallurgica 1988;22:847. 
[10] Crawford DC, Was GS. Metall. Trans. A 1992;23:1195.

[11] Dave VR, Cola MJ, Kumar M, Schwartz AJ, Hussen GNA. Welding Journal 2004;83:1S.

[12] Gao Y, Kumar M, Nalla RK, Ritchie RO. Metall. Mater. Trans. A 2005;36A:3325.

[13] Was GS, Alexandreanu B, Andresen P, Kumar M. Mater. Res. Soc. Proc. 2004;819:87.

[14] Kumar M, King WE, Schwartz AJ. Acta Mater. 2000;48:2081.

[15] Schuh CA, Kumar M, King WE. Acta Mater. 2003;51:687.

[16] Schuh CA, Minich RW, Kumar M. Philos. Mag. 2003;83:711.

[17] Reed BW, Kumar M, Minich RW, Rudd RE. Acta Mater. 2008;56:3278.

[18] Brandon DG, Ralph B, Ranganathan S, Wald MS. Acta Metall. 1964;12:813.

[19] Schwartz AJ, Kumar M, Adams BL, editors. Electron Backscatter Diffraction in Materials Science. Kluwer Academic/Plenum Publishers, New York, NY 2000.

[20] Kumar M, Schwartz AJ, King WE. Acta Mater. 2002;50:2599.

[21] Lassila DH, Birnbaum HK. Acta Metall. 1986;34:1237.

[22] Robertson WM. Metall. Trans. A 1979;10:489.

[23] Louthan MR, Donovan JA, Caskey GR. Acta Metall. 1975;23:745.

[24] Marchi CS, Somerday BP, Robinson SL. Intl. J. Hydrogen Energy 2007;32:100.

[25] ASTM E8M-98, in Annual Book of ASTM Standards, Vol. 03.01: Metals - Mechanical Testing; Elevated and Low-temperature Tests; Metallography. ASTM International, West Conshohocken, PA, USA 1998.

[26] Lassila DH, Birnbaum HK. Acta Metall. 1987;35:1815.

[27] ASTM E1820-06 in Annual Book of ASTM Standards, Vol. 03.01: Metals - Mechanical Testing; Elevated and Low-temperature Tests; Metallography. ASTM International, West Conshohocken, PA, USA 2006.

[28] Haggag FM, Underwood JH. Int. J. Fract. 1984;26:R63.

[29] Marchi CS, Somerday BP, Tang X, Schiroky GH. Intl. J. Hydrogen Energy 2008;33:889.

[30] Lassila DH, Birnbaum HK. Acta Metall. 1988;36:2821.

[31] Oriani RA. Acta Metall. 1970;18:147. 
Table 1. Microstructural conditions and mechanical properties of the two microstructures in their thermomechanically-processed conditions.

\begin{tabular}{lccc}
\hline Properties & $\begin{array}{c}\text { Low Special Fraction } \\
\text { Microstructure }\end{array}$ & $\begin{array}{c}\text { High Special Fraction } \\
\text { Microstructure }\end{array}$ \\
\hline \multicolumn{2}{l}{$\begin{array}{l}\text { Average grain } \\
\text { spize }(\mu \mathrm{m})\end{array}$} & $30.4 \pm 1.5$ & $30.6 \pm 1.5$ \\
$\begin{array}{l}\text { Special } \\
\text { boundary } \\
\text { fraction }\end{array}$ & Length & $0.46 \pm 0.02$ & $0.75 \pm 0.04$ \\
\cline { 2 - 4 } $\begin{array}{l}\text { Twin } \\
\text { boundary } \\
\text { fraction }\end{array}$ & Length & $0.35 \pm 0.02$ & $0.62 \pm 0.03$ \\
\cline { 2 - 4 } $\begin{array}{l}\text { 0.2\% offset yield strength }(\mathrm{MPa}) \\
\text { Ultimate tensile strength }(\mathrm{MPa})\end{array}$ & $0.38 \pm 0.02$ & $0.59 \pm 0.03$ \\
\hline
\end{tabular}

Table 2. Target lattice hydrogen concentrations $\left(C_{L}\right)$, hydrogen-charging temperatures and gas pressures, and measured total hydrogen concentrations $\left(C_{t o t}\right)$ for tensile and single edge-notched bend specimens.

\begin{tabular}{cccccc}
\hline Specimen Type & Specimen Set & $\begin{array}{c}\text { Target } C_{L} \\
(\mathbf{a p p m})\end{array}$ & $\begin{array}{c}\text { Temperature } \\
\left({ }^{(} \mathbf{C}\right)\end{array}$ & $\begin{array}{c}\mathbf{H}_{2} \text { Pressure } \\
(\mathbf{M P a})\end{array}$ & $\begin{array}{c}\text { Measured } C_{\text {tot }} \\
(\mathbf{a p p m})\end{array}$ \\
\hline \multirow{3}{*}{ Tensile } & 1 & 1000 & 150 & 55 & 1200 \\
\cline { 2 - 6 } & 2 & 2000 & 150 & 138 & 2200 \\
\hline \multirow{2}{*}{ Bend } & 3 & 3000 & 200 & 138 & 3400 \\
\cline { 2 - 6 } & 1 & 2000 & 200 & 83 & 2300 \\
\hline & 2 & 2000 & 200 & 83 & 2900 \\
\hline
\end{tabular}




\section{LIST OF FIGURE CAPTIONS}

Fig. 1. EBSD orientation maps of the grain structures in Ni-201 of the microstructures with a (A) low fraction ( $46 \%$ by length) and (B) high fraction ( $75 \%$ by length) of special grain boundaries. Random boundaries are depicted in black, $\Sigma 3$ twin boundaries in red, and other $\Sigma 3^{n}$ special boundaries in blue. Grain size and texture remain essentially unchanged.

Fig. 2. Normalized reduction in area, $R A / R A$, plotted as a function of hydrogen concentration for the two Ni-201 microstructures with high (75\% by length) and low ( $46 \%$ by length) fractions of special boundaries. The reduction in area of the charged material, RA, was measured after tensile testing, and normalized by the reduction in area in the uncharged condition, RA. The severity of embrittlement is markedly reduced by increasing the fraction of special grain boundaries.

Fig. 3. Fraction of brittle intergranular cracking on the fracture surfaces of the uniaxial tensile specimens as a function of the dissolved hydrogen concentration. The increase in fraction of special grain boundaries clearly decreases the proportion of intergranular fracture within the material.

Fig. 4. Secondary electron micrographs of fracture surfaces after tensile testing. (A) In their uncharged conditions, both "low" and "high" special boundary microstructure display dimpled fracture surfaces that are typical of microvoid coalescence observed in ductile materials. (B, C) After hydrogen charging at 3400 appm, (B) the "low special boundary" microstructure show exclusively intergranular fracture typical of hydrogen embrittled structures. (C) In contrast, "high special fraction" microstructures lower the fraction of intergranular cracking significantly. 
Fig. 5. J-R $(\Delta a)$ resistance-curves for stable crack extension in grain-boundary engineered Ni-201 containing total hydrogen concentrations, $C_{t o t}$ of (A) 2300 appm and (B) 2900 appm. Each data point represents a partial unloading event during the test. The results clearly indicate that an increase in the fraction of special grain boundaries results in an increase in both the (crack-initiation) fracture toughness, $J_{c}$ and the extent of subcritical crack growth prior to catastrophic failure.

Fig. 6. Secondary electron micrographs of fracture surfaces after R-curve testing for a total hydrogen concentration, $C_{\text {tot, }}$ of 2300 appm. (A) The low special fraction microstructure shows $100 \%$ intergranular fracture, while (B) the high special fraction microstructure displays a more ductile fracture mode.

Fig. 7. Secondary electron micrograph of fracture surface from hydrogen-charged Nickel-201 after tensile testing. The intergranular fracture facets show evidence of slip traces. 

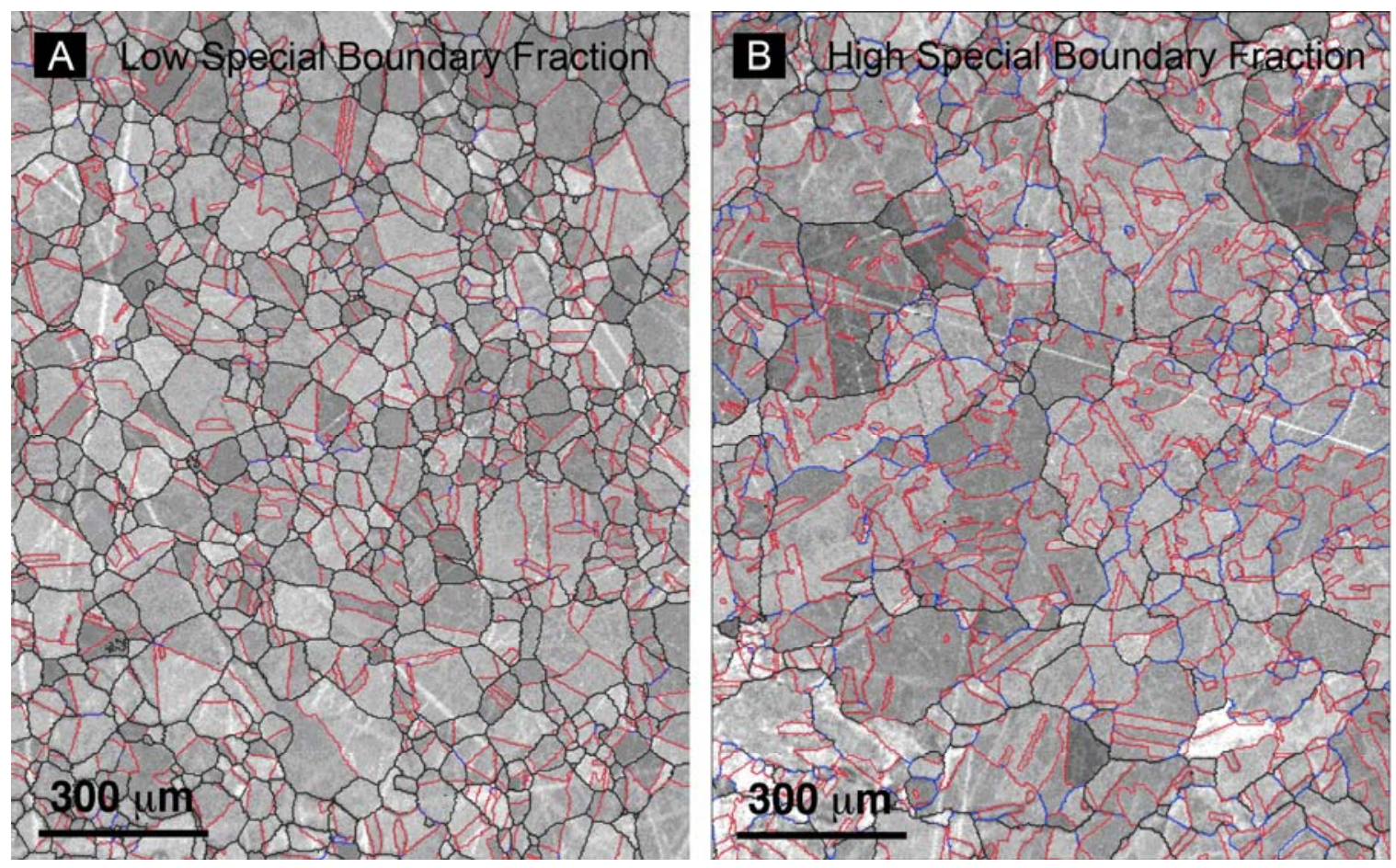

Fig. 1. EBSD orientation maps of the grain structures in Ni-201 of the microstructures with a (A) low fraction (46\% by length) and (B) high fraction (75\% by length) of special grain boundaries. Random boundaries are depicted in black, $\Sigma 3$ twin boundaries in red, and other $\Sigma 3^{n}$ special boundaries in blue. Grain size and texture remain essentially unchanged. 


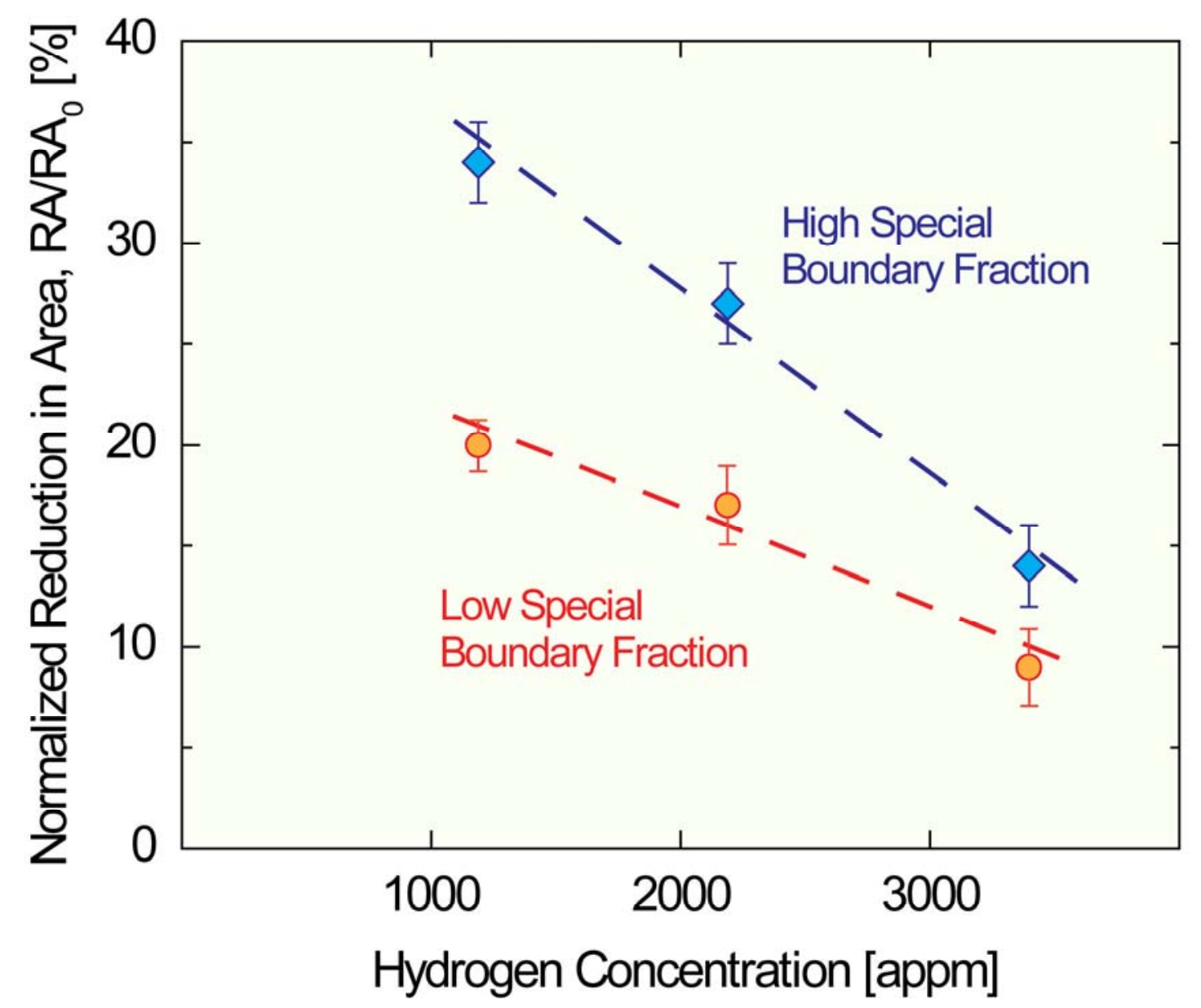

Fig. 2. Normalized reduction in area, $R A / R A$, plotted as a function of hydrogen concentration for the two Ni-201 microstructures with high (75\% by length) and low ( $46 \%$ by length) fractions of special boundaries. The reduction in area of the charged material, RA, was measured after tensile testing, and normalized by the reduction in area in the uncharged condition, RA. The severity of embrittlement is markedly reduced by increasing the fraction of special grain boundaries. 


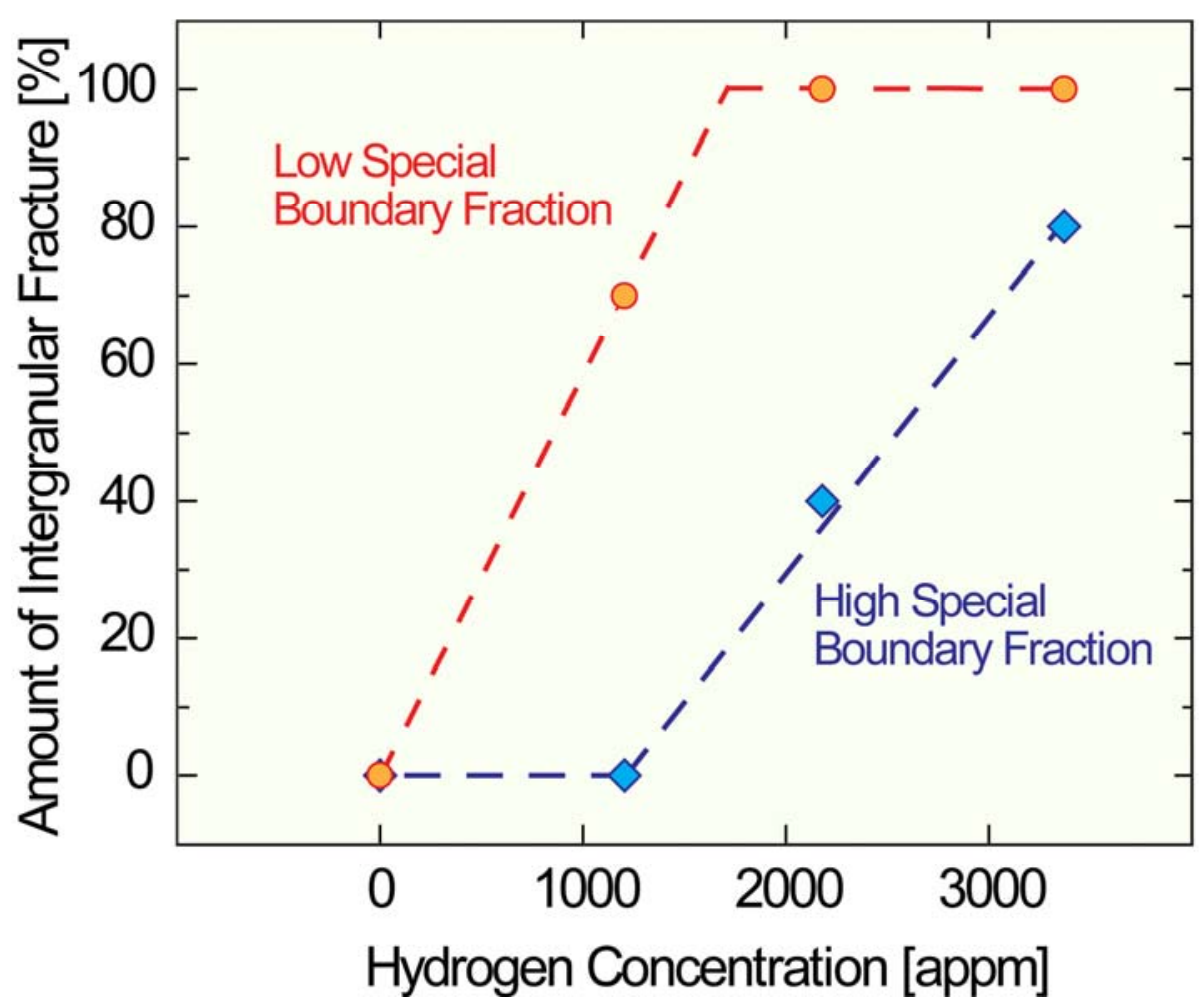

Fig. 3. Fraction of brittle intergranular cracking on the fracture surfaces of the uniaxial tensile specimens as a function of the dissolved hydrogen concentration. The increase in fraction of special grain boundaries clearly decreases the proportion of intergranular fracture within the material. 

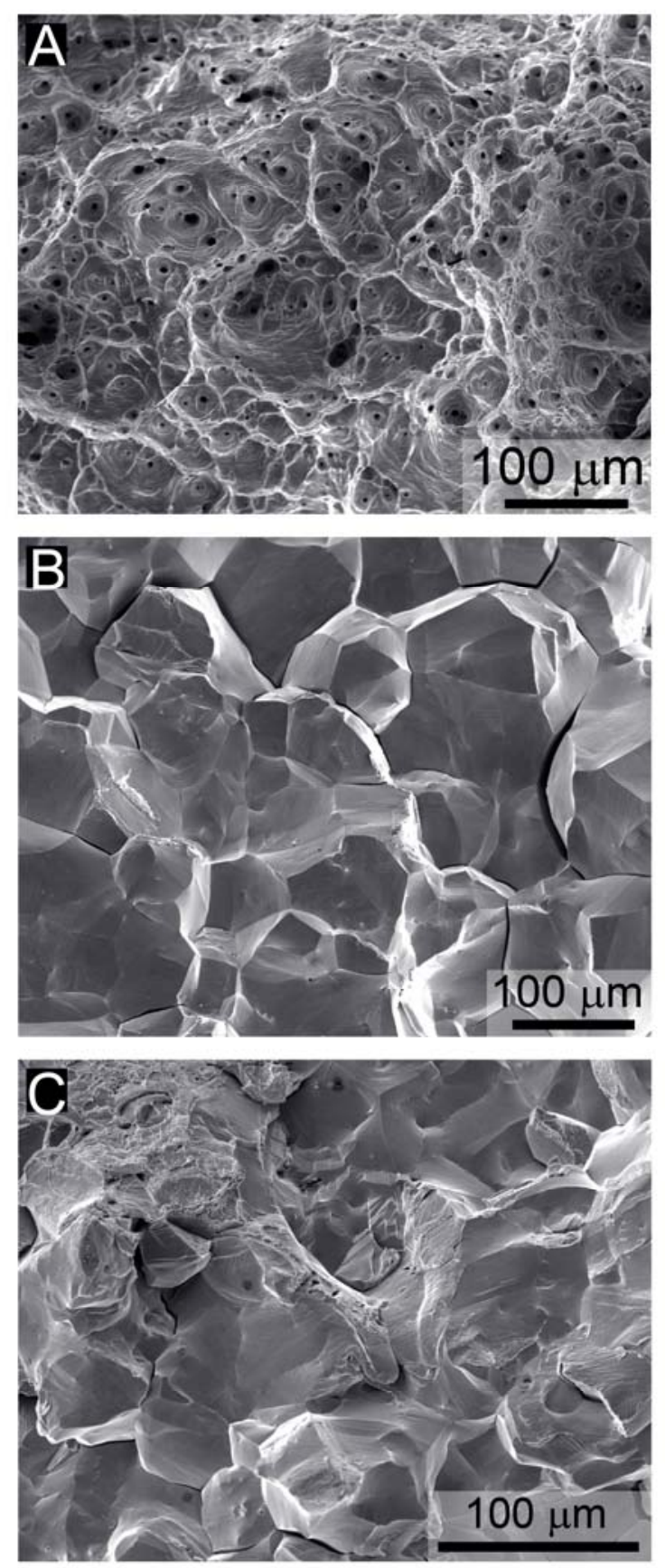

Fig. 4. Secondary electron micrographs of fracture surfaces after tensile testing. (A) In their uncharged conditions, both "low" and "high" special boundary microstructure display dimpled fracture surfaces that are typical of microvoid coalescence observed in ductile materials. (B, C) After hydrogen charging at 3400 appm, (B) the "low special boundary" microstructure show exclusively intergranular fracture typical of hydrogen embrittled structures. (C) In contrast, "high special fraction" microstructures lower the fraction of intergranular cracking significantly. 

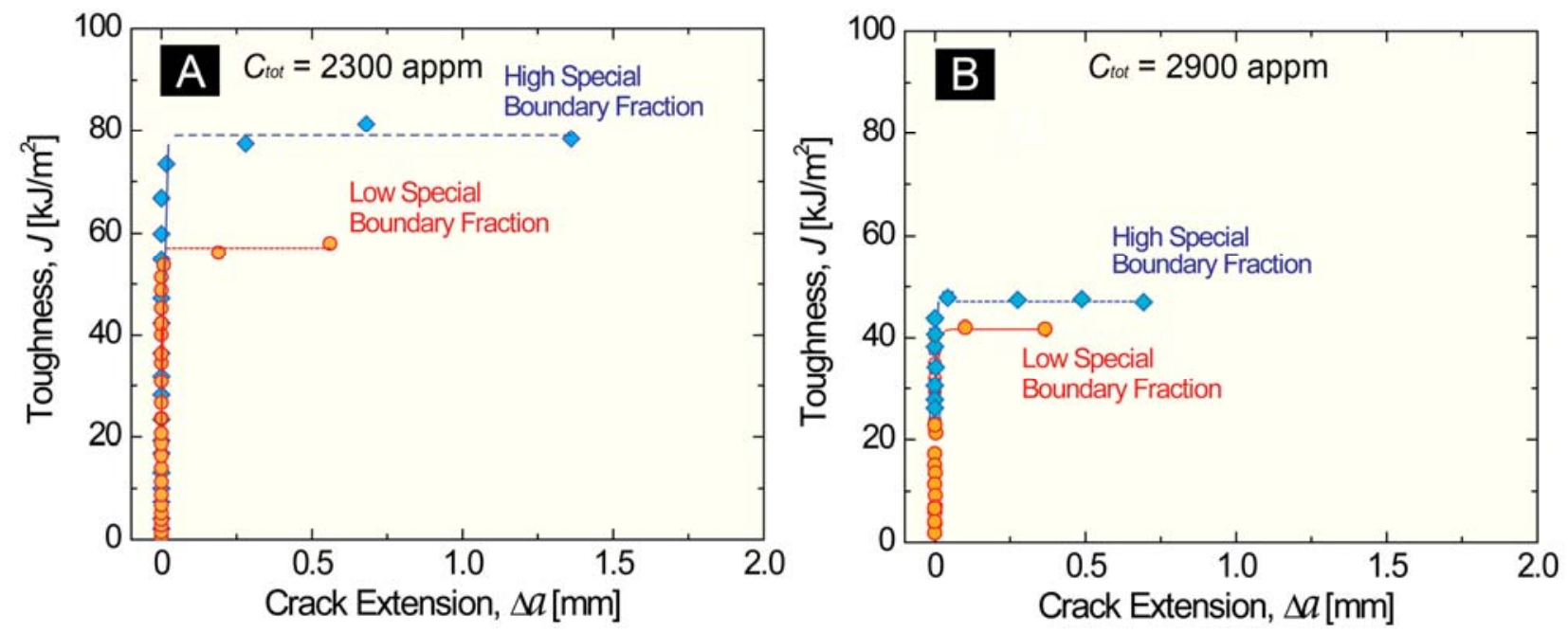

Fig. 5. J-R $(\Delta a)$ resistance-curves for stable crack extension in grain-boundary engineered Ni-201 containing total hydrogen concentrations, $C_{\text {tot, }}$ of (A) $2300 \mathrm{appm}$ and (B) 2900 appm. Each data point represents a partial unloading event during the test. The results clearly indicate that an increase in the fraction of special grain boundaries results in an increase in both the (crack-initiation) fracture toughness, $J_{c}$ and the extent of subcritical crack growth prior to catastrophic failure. 

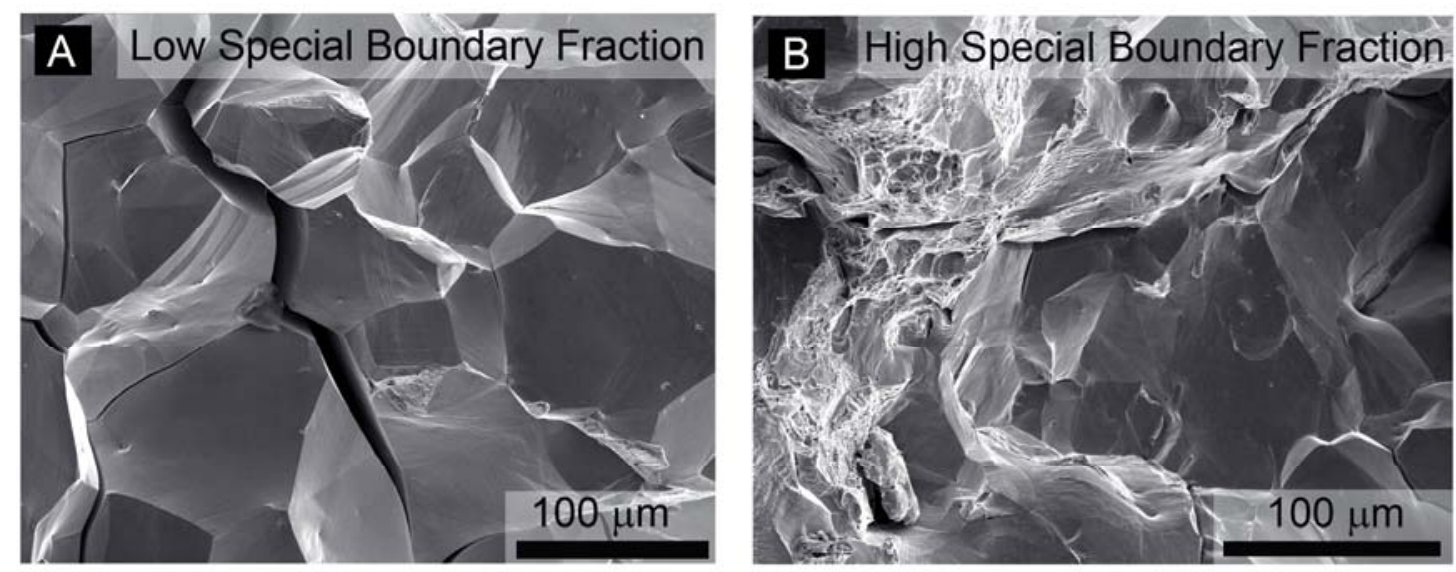

Fig. 6. Secondary electron micrographs of fracture surfaces after R-curve testing for a total hydrogen concentration, $C_{t o t}$ of 2300 appm. (A) The low special fraction microstructure shows $100 \%$ intergranular fracture, while (B) the high special fraction microstructure displays a more ductile fracture mode. 


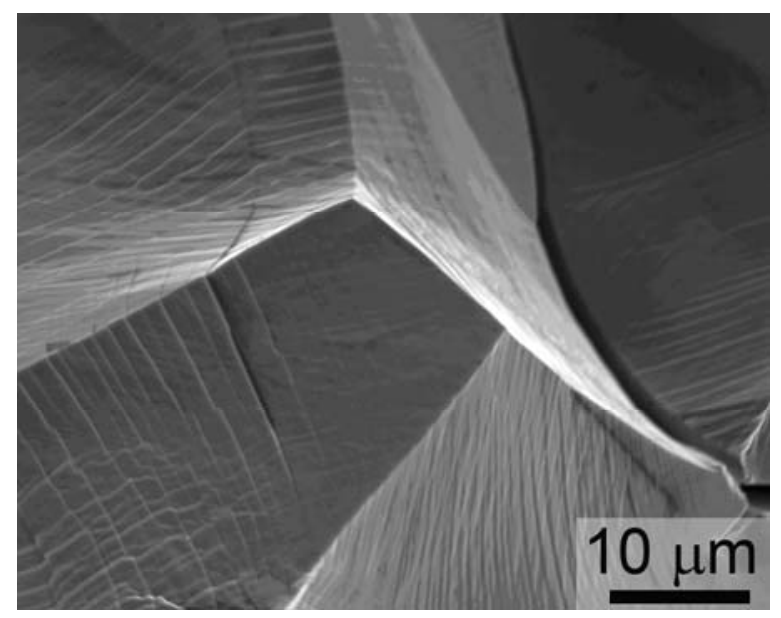

Fig. 7. Secondary electron micrograph of fracture surface from hydrogen-charged Nickel-201 after tensile testing. The intergranular fracture facets show evidence of slip traces. 